\title{
Genetic control of compound leaf development in the mungbean (Vigna radiata $\mathrm{L}$.)
}

Keyuan Jiao', Xin Li $\mathbb{1}^{2}$, Shihao Su $\mathbb{0}^{3}$, Wuxiu Guo ${ }^{3}$, Yafang Guo², Yining Guan², Zhubing Hư ${ }^{4}$, Zhenguo Shen ${ }^{2}$ and Da LuO $^{3}$

\begin{abstract}
Many studies suggest that there are distinct regulatory processes controlling compound leaf development in different clades of legumes. Loss of function of the LEAFY (LFY) orthologs results in a reduction of leaf complexity to different degrees in inverted repeat-lacking clade (IRLC) and non-IRLC species. To further understand the role of $L F Y$ orthologs and the molecular mechanism in compound leaf development in non-IRLC plants, we studied leaf development in unifoliate leaf (un) mutant, a classical mutant of mungbean (Vigna radiata L.), which showed a complete conversion of compound leaves into simple leaves. Our analysis revealed that UN encoded the mungbean LFY ortholog (VrLFY) and played a significant role in leaf development. In situ RNA hybridization results showed that STM-like KNOXI genes were expressed in compound leaf primordia in mungbean. Furthermore, increased leaflet number in heptafoliate leaflets 1 (hel1) mutants was demonstrated to depend on the function of VrLFY and KNOXI genes in mungbean. Our results suggested that HEL1 is a key factor coordinating distinct processes in the control of compound leaf development in mungbean and its related non-IRLC legumes.
\end{abstract}

\section{Introduction}

Plant leaves are the primary photosynthetic organs that are initiated on the flanks of the shoot apical meristem (SAM). The class I KNOTTED1-like homeobox (KNOXI) genes are involved in the maintenance of the meristem activity of SAM, while the initiation of leaves requires downregulation of KNOXI genes at the incipient site ${ }^{1-3}$. In simple-leafed species such as Arabidopsis thaliana, downregulation of KNOXI genes in leaf primordia is permanent, whereas in most compound-leafed eudicot species, including the tomato (Solanum lycopersicum) and Cardamine hirsuta, KNOXI genes are reactivated in leaf primordia after initiation of leaf development ${ }^{4-6}$. In $C$.

\footnotetext{
Correspondence: Xin Li (lixin@njau.edu.cn)

${ }^{1}$ Institute of Traditional Chinese Medicine and Natural Products, College of

Pharmacy, Jinan University, Guangzhou, China

${ }^{2}$ College of Life Sciences, Laboratory Center of Life Sciences, Nanjing

Agricultural University, Nanjing, China

Full list of author information is available at the end of the article.

These authors have contributed equally: Keyuan Jiao, Xin Li and Shihao Su
}

hirsuta, the leaflet number is reduced in mutants of the KNOXI gene SHOOTMERISTEMLESS (ChSTM) or BREVIPEDICELLUS $(\mathrm{ChBP})^{7,8}$. In S. lycopersicum, ectopic expression of the KNOXI genes Tomato KNOTTED1 (Tkn1) or Tkn2 (orthologs of STM and BP in tomato, respectively) in transgenic lines, or upregulated expression of $T k n 1$ or $T k n 2$ in related mutants, results in ramification for compound leaves suggesting that regulatory processes mediated by KNOXI genes, especially $S T M / B P$-like KNOXI genes, play pivotal roles in compound leaf development ${ }^{5,9,10}$.

However, in the inverted repeat-lacking clade (IRLC) of legumes, which includes Pisum sativum and Medicago truncatula, the expression of STM/BP-like KNOXI genes is excluded from leaf primordia ${ }^{11-13}$. Genetic analysis shows that single mutants, double mutants and triple mutants of $3 S T M / B P$-like KNOXI genes, namely, $M t K N O X 1, M t K N O X 2$, and MtKNOX6, in M. truncatula do not show obvious defects in compound leaves ${ }^{13}$. Thus,

\section{(c) The Author(s) 2019}

(c) (i) Open Access This article is licensed under a Creative Commons Attribution 4.0 International License, which permits use, sharing, adaptation, distribution and reproduction c. in any medium or format, as long as you give appropriate credit to the original author(s) and the source, provide a link to the Creative Commons license, and indicate if changes were made. The images or other third party material in this article are included in the article's Creative Commons license, unless indicated otherwise in a credit line to the material. If material is not included in the article's Creative Commons license and your intended use is not permitted by statutory regulation or exceeds the permitted use, you will need to obtain permission directly from the copyright holder. To view a copy of this license, visit http://creativecommons.org/licenses/by/4.0/. 
$S T M / B P$-like KNOXI genes may not be involved in compound leaf development in IRLC legume plants ${ }^{11-13}$. Instead, another type of transcription factor, UNIFOLIATA (UNI) in P. sativum and SINGLE LEAFLET1 (SGL1) in M. truncatula, orthologs of LEAFY (LFY) from Arabidopsis, functions in controlling compound leaf development ${ }^{14-16}$. The uni mutants in pea and sgl1 mutants in $M$. truncatula exhibit single leaflet phenotypes, and inflorescence and floral defects ${ }^{15,16}$. Hence, the LFY orthologs appear to play a significant role in compound leaf development in IRLC legumes. Furthermore, it has been shown that the UNI cofactor UNUSUAL FLORAL ORGANS (UFO) in pea, and PALM1, an upstream transcription factor of SGL1 in M. truncatula, are involved in the control of leaf complexity ${ }^{17,18}$. Recent studies show that the adaxial-abaxial regulators PHANTASTICA (PHAN), ARGONAUTE7 (AGO7), and AUXIN RESPONSIVE FACTOR 3 (ARF3) regulate the expression level of PALM1 and therefore control compound leaf development in M. truncatula ${ }^{19-21}$.

The function of the $L F Y$ orthologs during compound leaf development has also been investigated in nonIRLC legumes, including soybean and L. japonicus in which KNOXI proteins are expressed in leaves, and are likely associated with compound leaf development ${ }^{12,22}$. In $L$. japonicus, a mutant of the $L F Y$ ortholog Proliferating Floral Meristem (PFM) exhibits one or two reduced basal leaflets ${ }^{12}$. In soybean $L F Y$-RNAi-silenced lines, only the leaflet number of the compound leaves produced at the second node is reduced ${ }^{12}$. This would indicate that there is a minor role in compound leaf development for $L F Y$ orthologs in non-IRLC legume species $^{12,22,23}$.

In this study, we described the compound leaf developmental processes in a non-IRLC legume species, mungbean (Vigna radiata L.), a fast-growing (60-90 days) warm-season grain legume, and characterized the unifoliate leaf (un) mutants that showed a complete conversion of compound leaves into simple leaves. Four alleles of un carried mutations in the $L F Y$ ortholog, indicating that the $L F Y$ ortholog in mungbean played a significant, rather than a minor role in compound leaf development. Phylogenetic analysis of the KNOX gene family in legumes was conducted, and the expression of four STM/BP-like KNOXI genes was characterized in mungbean using in situ RNA hybridization. Furthermore, genetic interaction and gene expression analysis showed that increased leaflet number in heptafoliate leaflets1 (hel1) mutants involved regulatory processes mediated by $V r L F Y$ and $S T M / B P$-like KNOXI genes in mungbean. This study showed that the LFY ortholog might play a more significant role in the control of compound leaf development earlier than the time estimated by Champagne et al. ${ }^{12}$.

\section{Materials and methods}

Plant material and growth conditions

All the mutants were isolated from the $\mathrm{M}_{2}$ population of a mutagenized mungbean cultivar, Sulu, generated in Nanjing, China. The gamma irradiator was calibrated to irradiate the seed lots with $400 \mathrm{~Gy}$ of gamma rays. The $M_{1}$ seeds were sown in the field, and the $M_{2}$ seeds were individually harvested from the population. Approximately 36 seeds of each $\mathrm{M}_{2}$ line were planted in individual rows in the field, with a distance of $0.3 \mathrm{~m}$ between rows. The mutant plants were then individually harvested and sown for further observation in the greenhouse at $26-30{ }^{\circ} \mathrm{C}$ with a 16 -h light/8-h dark photoperiod at $200 \mu \mathrm{mol} \mathrm{m}^{-2} \mathrm{~s}^{-1}$. The allelic nature of genes was confirmed by crosses among un1-1, un1-2, un1-3, and un1-4, using heterozygote parents because the mutants were sterile (the mutant plants were found in $F_{1}$ plants of the crosses). The L. japonicus ecotype Gifu B-129 was grown at $20-22{ }^{\circ} \mathrm{C}$ with a 16 -h light/8-h dark photoperiod at $150 \mu \mathrm{mol} \mathrm{m}^{-2} \mathrm{~s}^{-1}$ in the greenhouse.

\section{Scanning electron microscopy}

Mungbean shoot apices at different developmental stages were fixed in FAA solution containing 3.7\% (v/v) formaldehyde, $50 \%(\mathrm{v} / \mathrm{v})$ ethanol, and $5 \%(\mathrm{v} / \mathrm{v})$ acetic acid. Before vacuum freeze drying, fixed samples were dehydrated in an ethanol/tert-butanol series. The materials were transferred to a vacuum freeze dryer overnight. The preparation of shoot apices for scanning electron microscopy (SEM) analysis was as described by Chen et al. ${ }^{24}$. Samples were examined in a JEOL JSM-6360LV (JEOL) SEM at $10-15 \mathrm{kV}$ of acceleration voltage.

\section{Molecular cloning of the full-length $L F Y$ gene (VrLFY) from mungbean}

To clone the full-length mungbean $L F Y$ gene, we first searched the genome sequence database of mungbean (http://plantgenomics.snu.ac.kr) using sequences for $U N I$ and SGL1. Finally, sequence alignment with $U N I / S G L 1 /$ $L F Y / F L O$ coding sequences allowed the open reading frame of $V r L F Y$ to be defined. Polymerase chain reaction (PCR) was carried out using the primers in Supplementary Table 3. PCR products were cloned into the pGEM-T easy vector (Promega), and inserts were characterized by nucleotide sequencing.

\section{In situ hybridization}

For in situ probes, gene-specific regions of $\operatorname{VrLFY}$, VrKNOXI, and LjKNOXI genes were generated by PCR with primer sets (see Supplemental Table 4) and cloned into a pGEM-T vector (Promega, A1360). In situ probes were synthesized from these clones by in vitro transcription using the Digoxigenin RNA Labeling Kit (Roche) from either the T7 or SP6 promoter flanking the 
insert, generating either sense or anti-sense probes. Mungbean shoot apices were fixed overnight in $4 \%$ (wt/vol) paraformaldehyde, $\mathrm{pH} 7.0$, and then embedded in Paraplast (Sigma). RNase-free slices of the shoot apices were hybridized to digoxigenin-labeled probes and used for subsequent immunological detection as previously described $^{25}$.

\section{Expression analysis by quantitative reverse transcription PCR}

Shoot apices from 2-week-old mutant and wild-type plants were collected in RNase-free tubes and stored in a $-80^{\circ} \mathrm{C}$ freezer. Samples were taken in triplicate as biological replicates. Total RNA was extracted using the Plant RNA Kit (Omega) following the manufacturer's instructions. Samples were then treated with RNase-free DNase I (Promega) for $30 \mathrm{~min}$.

For quantitative reverse transcription PCR (qRT-PCR), first-strand cDNA was synthesized from total RNA using the First Strand cDNA Synthesis Kit (Fermentas). Realtime RT-PCR analysis was performed as three technical replicates in 384-well plates using SYBR $^{\circledast}$ Premix ExTaq (Takara), on an ABI StepOnePlus machine, according to the manufacturer's protocol (Applied Biosystems). The relative expression level of genes was determined by the $2^{-\triangle \Delta C T}$ method. Amplification of VrTUB ( $\mathrm{Vra}$ di05g13910), a constitutively expressed gene, was used as an internal control to normalize all data ${ }^{26}$. Shoot apices from a single genotype were represented by nine samples; independent total RNA isolations were generated from the three biological replicates, and three technical qRTPCR replicates were performed on each of the total RNA preparations. The primers used for qRT-PCR are given in Supplementary Table 5.

\section{Transcript profiling by deep-sequencing}

For Illumina sequencing, mRNA was purified from shoot apices of 2-week-old seedlings of un 1-1 mutant and wild-type plants and then fragmented into small pieces. Random hexamer primers and reverse transcriptase (Invitrogen) were used to carry out first-strand cDNA synthesis. Second-strand cDNA synthesis was performed with DNA polymerase I (New England BioLabs) after RNase $\mathrm{H}$ (Invitrogen) treatment. Four cDNA libraries were constructed, and cDNA sequencing was conducted using the Illumina HiSeq X Ten system according to the manufacturer's protocol, with read lengths of $150 \mathrm{bp}$. The raw data were submitted to the NCBI Short Read Archive (SRP110723). The number of reads per kilobase of exon region in a gene per million mapped reads (RPKM) was used to normalize the gene expression data ${ }^{27,28}$. Differentially expressed genes were determined between the wild type and mutants according to statistical analysis of the frequency of each transcript, and their corresponding
P-values were calculated ${ }^{25}$. The significance threshold of $P$-values in multiple tests was set by the false discovery rate (FDR). We used a FDR $\leq 0.05$ and an absolute value of $\mid \log _{2}$ ratio $\mid \geq 1$ as the threshold to judge the significance of gene expression differences.

\section{Phylogenetic analysis}

The phylogenetic tree was constructed using the Molecular Evolutionary Genetics Analysis software (MEGA; version 6.0) by the neighbor-joining method (JFF Matrix model) with 1000 bootstrap replications.

\section{Data availability}

Sequence data from this article can be found in the GenBank data libraries under the following accession numbers: XP_014491863.1 (VrLFY); XP_017441945.1 (VaLFY); XP_007137848.1 (PhvLFY); XP_003526918.2 (GmLFY1); XP_014630701.1 (GmLFY2); AAX13294.1 (PFM); AAC49782.1 (UNI); XP_003602745.1 (SGL1); XP_002284664.1 (VFL); AF197934_1 (FALSIFLORA); AAM27941.1 (LEAFY); AAA62574.1 (FLORICAULA); AQQ16908.1 (ChLFY); XP_015635355.1 (RFL); and O04407.1 (NEEDLY).

\section{Results \\ Compound leaf development in mungbean}

Similar to M. truncatula, L. japonicus, and other compound leaf-producing species, leaf development in mungbean was heteroblastic. The first pair of juvenile leaves with simple leaf morphologies emerged in opposite phyllotaxy on the first node of a developing mungbean plant and was succeeded by adult trifoliate leaves in alternate phyllotaxy (Fig. 1a-c). The wild-type trifoliate leaves of mungbean consisted of a pair of stipules, a petiole, 2 lateral leaflets, a rachis, and a single terminal leaflet (Fig. 1c). To facilitate the characterization of leaf mutants in mungbean, we investigated leaf developmental processes by scanning electron microscopy (SEM). The morphological changes during compound leaf development in mungbean can be divided into seven distinct stages. At Stage $0(\mathrm{~S} 0)$, cells along the periphery of SAM were recruited as founder cells and became an incipient leaf primordium (Fig. 1d). At S1, a common leaf primordium formed as a strip of cells grew out along the periphery of SAM (Fig. 1d). At the subsequent S2, a pair of stipule primordia emerged at the proximal end of the common leaf primordium (Fig. 1e), enlarged, and then separated away from the common leaf primordium so that boundaries were established between the two stipules and the common leaf primordium at S3 (Fig. 1f). At S4, a pair of lateral leaflet primordia emerged between the stipule and the common leaf primordium; and then the common leaf primordium differentiated into a terminal leaflet primordium (Fig. 1g). 

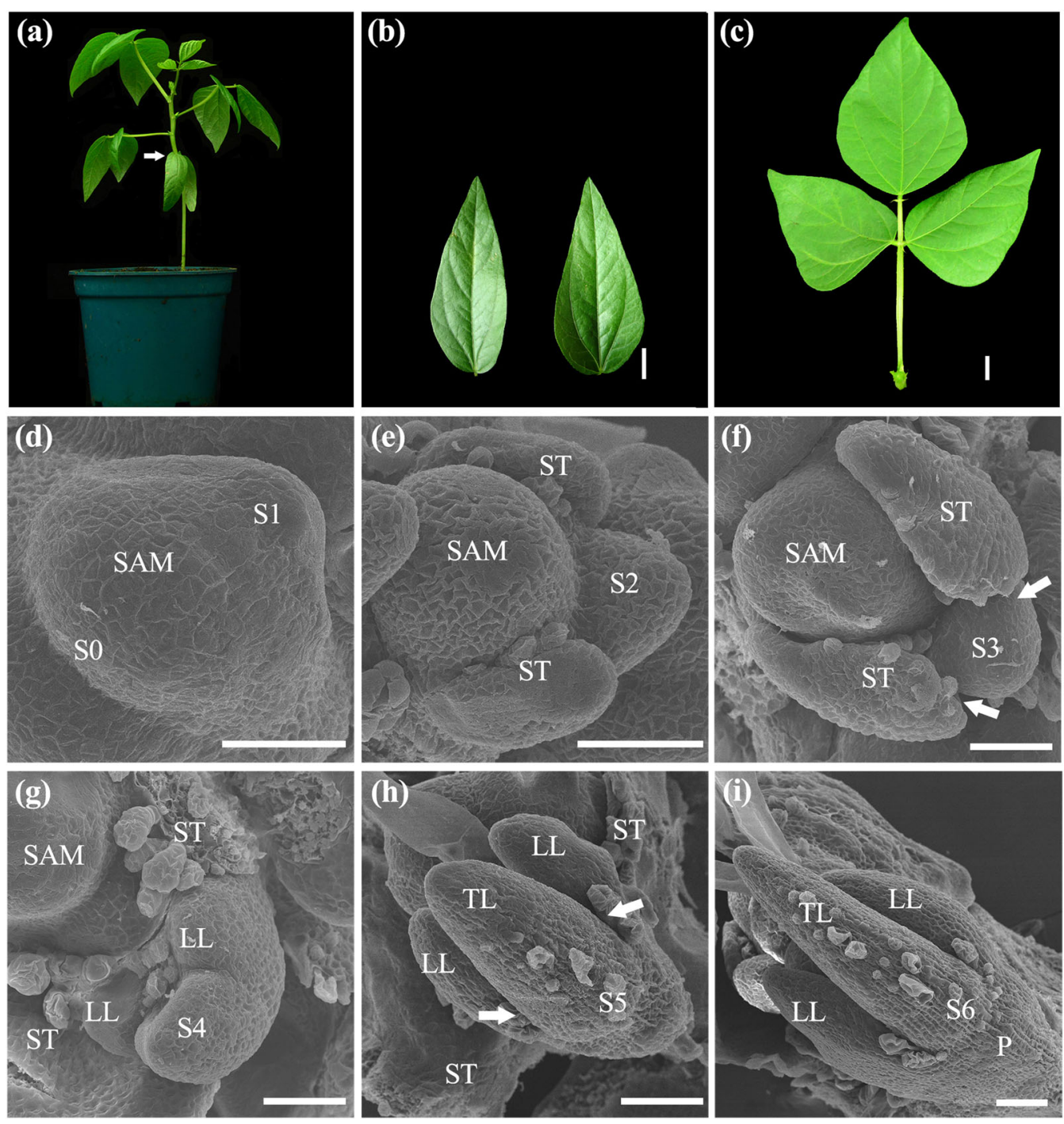

Fig. 1 The ontogeny of compound leaf development in wild-type mungbean. a Whole plant morphology of mungbean. The arrow indicates the opposite juvenile leaves at the first node. $\mathbf{b}$ A pair of juvenile leaves of mungbean. $\mathbf{c}$ Adult compound leaf of mungbean. $\mathbf{d}-\mathbf{i}$ SEM analysis of compound leaf development. $\mathbf{d}$ Sites of the incipient leaf primordia were specified at the periphery of the SAM at S0. At S1, a common leaf primordium was initiated as a strip of cells outgrowing along the periphery of SAM. e A pair of stipule primordia (ST) was initiated from the proximal end of the common leaf primordium at S2. $\mathbf{f}$ At S3, the boundaries (arrows) between the stipule and lateral leaflet primordia were formed. $\mathbf{g}$ At S4, a pair of lateral leaflet primordia (LL) emerged between the stipule and common leaf primordium. $\mathbf{h}$ At $\mathbf{S}$, the common leaf primordium differentiated into a terminal leaflet primordium (TL) as indicated by development of trichomes from the abaxial surface. Boundaries (arrows) were formed between the lateral and terminal leaflet primordia. i At S6, the leaflet primordia folded as a result of outgrowth of the abaxial surface, and the region between the stipule and lateral leaflet primordia expanded to form a petiole (P). Trichomes developed from the abaxial surface of both the stipule and lateral leaflet primordia. b, c Scale bars $=1 \mathrm{~cm}$; $\mathbf{d}-\mathbf{i}$ Scale bars $=50 \mu \mathrm{m}$

Subsequently, at S5, the lateral leaflet primordia were separated away from a terminal leaflet primordium so that a boundary was established, and trichomes initiated from the abaxial surface of the terminal leaflet primordium (Fig. 1h). Following S5, the lateral leaflets and terminal leaflet primordium became folded (Fig. 1i), and the region between the stipule and lateral leaflet primordia expanded to become a petiole as a result of cell division and cell expansion at the S6 stage.
Isolation and characterization of un mutants in mungbean

Four similar leaf mutants (Supplementary Table 1), which mimicked the phenotype of the classical mutant un, were isolated from a mutant population of mungbean generated by gamma irradiation ${ }^{29}$. Genetic analyses demonstrated that they were allelic (Materials and Methods section). In these mutants, all adult leaves consisted of a short petiole bearing a single terminal leaflet (Fig. 2a, b). Compared with the terminal leaflet of the wild type, the 

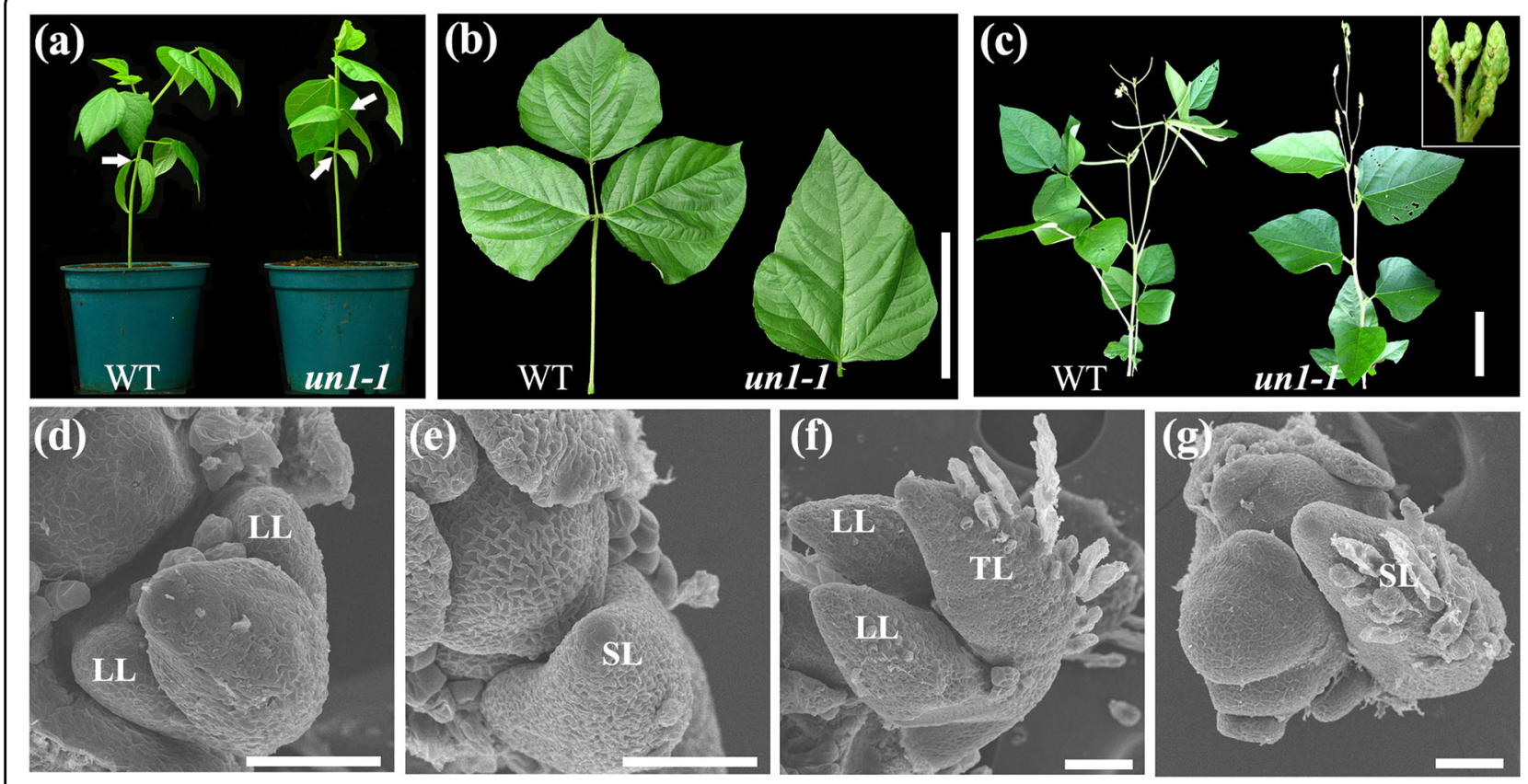

Fig. 2 Phenotypes of mungbean unifoliate (un) mutants. a Wild-type mungbean (left) and un 1-1 mutant (right) exhibiting compound and simple leaf forms, respectively. Arrows indicate the opposite juvenile leaves at the first node in the wild type and at the first and second nodes in the un 1-1 mutant. b Close-up views of the adult leaves of the wild type (left) and un 1-1 mutant (right). c Morphology of a mature un1-1 mutant (right), exhibiting simple leaf and floral homeotic phenotypes compared to the wild type (left). The inset has a close-up view of the inflorescence of the un 11 mutant. $\mathbf{d}$, $\mathbf{f}$ Leaf development of wild type at S4 and S6. e, $\mathbf{h}$ Leaf development of un $1-1$ at S4 and S6. The lateral leaflet primordia did not form at the proximal end of the common leaf primordium. LL lateral leaflet, TL terminal leaflet, SL single leaflet. b-c Scale bars $=10 \mathrm{~cm}$; d-f $50 \mu \mathrm{m}$ indicated

single terminal leaflet of the mungbean un mutant was larger (Fig. 2b). We found that the heteroblastic progression was also delayed in $u n$ mutants such that two simple leaves were produced at the first and second nodes in opposite phyllotaxis. Flowers that developed in $u n$ mutants were abnormal and infertile. un mutants produced sepallike proliferating structures that lacked petals and stamens, and the number of whorls of organs within the flowers was increased (Fig. 2c). Because of their infertility, un mutants were maintained as heterozygotes. Progeny from self-pollination of heterozygous lines segregated in a 3:1 ratio (33 wild-type plants and 9 mutant plants, $\chi^{2}=0.28<$ $\left.\chi_{0.05}^{2}=5.99\right)$, suggesting that the mutant phenotype was controlled by a single-recessive gene.

To characterize leaf development defects in mungbean un mutants, SEM analysis of leaf development was conducted. This analysis indicated that in $u n$ mutants, leaf development was initially normal until S4, at which point, the pair of lateral leaflet primordia failed to initiate between the stipule and the common leaf primordium (Fig. 2d, e). All four alleles of the mungbean un mutants exhibited this identical defect. The defect in the initiation of the lateral leaflet primordia was persistent throughout subsequent developmental stages, resulting in the formation of simple adult leaves in the un mutants of mungbean (Fig. 2f, g).

\section{Molecular cloning of $\operatorname{VrLFY}$ in mungbean}

The floral homeotic defects and single-leaf phenotype of the $u n$ mutants resembled that of the uni mutant in pea and the sgl1 mutant in $M$. truncatula ${ }^{15,16}$. The full-length DNA sequence of the $L F Y$ ortholog (VrLFY) in mungbean was obtained from the mungbean genomic database; the genomic sequence of $V r L F Y$ was $2155 \mathrm{bp}$ in length. Alignment of the genomic sequence of $V r L F Y$ with its cDNA sequence showed the existence of two introns (Supplementary Figure 1a). PCR amplification of mungbean genomic $L F Y$ from the $u n$ mutants and from the wild-type plants indicated that three un alleles (un1-1, un1-2, and un1-3) carried deletions (Fig. 3a). Nucleotide sequencing showed that another allele, the un1-4 mutant, had only a single base-pair substitution from the wild type gene (GAA to GGA, Supplementary Figure 1a). This resulted in an amino acid change (E112G, where the acidic amino acid Glu was replaced by a neutral amino acid Gly) in the $\mathrm{N}$ terminal domain of the protein (Supplementary Figure 1b).

Segregation analysis of an $\mathrm{F}_{2}$ population of the un1-1 allele indicated that 50 out of a total of 208 individuals were homozygous for the deletion and exhibited both simple leaf and floral homeotic defects, suggesting that the deletion in the corresponding $\operatorname{VrLFY}$ gene cosegregated with the mutant phenotype. Thus, the locus of 

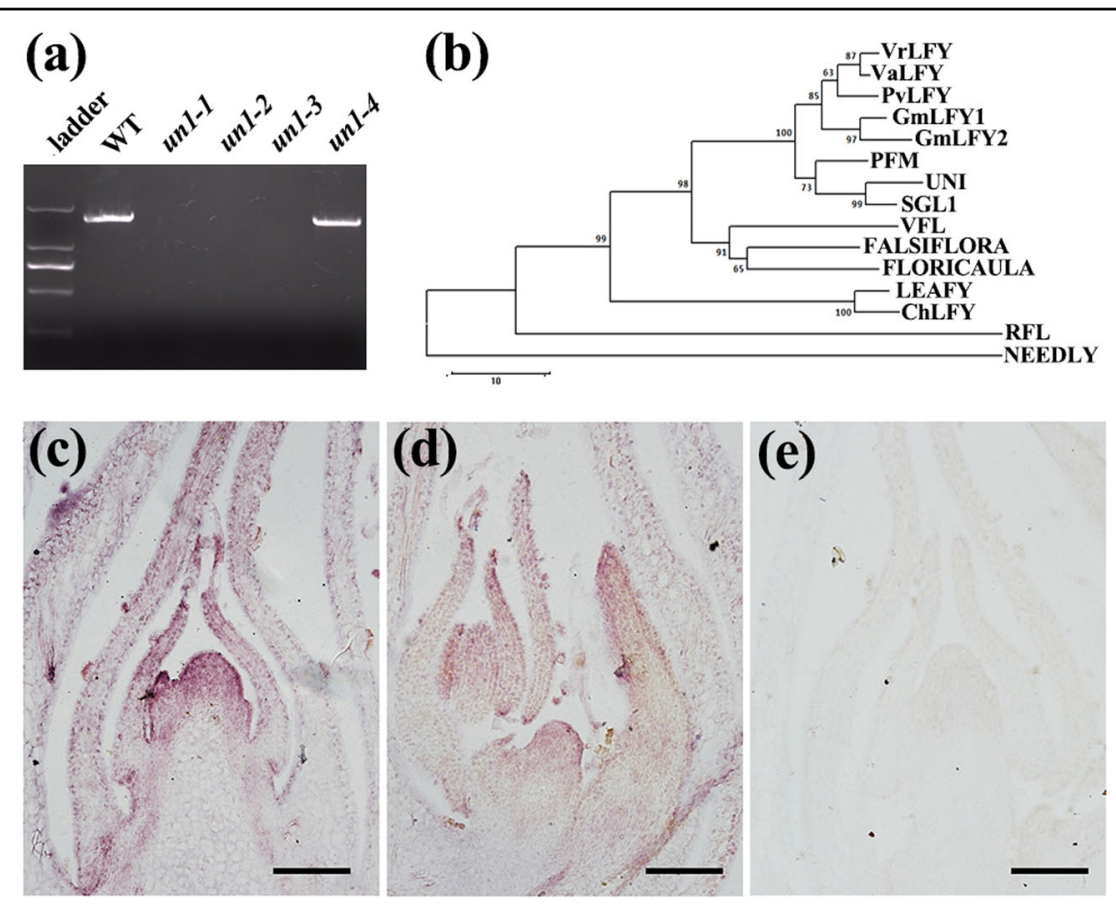

Fig. 3 Molecular cloning of the VrLFY gene from mungbean. a PCR amplification of the VrLFY gene from wild-type mungbean and un mutants (WT, un 1-1, un 1-2, un 1-3, and un 1-4). Deletions were detected as no product on attempted amplification of the VrLFY gene from three mutant alleles. b Phylogenetic analysis of VrLFY and its putative orthologs: VaLFY of $V$. angularis, PVLFY of Phaseolus vulgaris, GmLFY1 and GmLFY2 of soybean, PFM of L. japonicas, UNI of pea, SGL1 of M. truncatula, VFL of Vitis vinifera, FALSIFLORA of tomato, FLORICAULA of snapdragon, LEAFY of Arabidopsis, ChLFY of $C$. hirsute, RFL of rice and NEEDLY of Pinus radiata. Bootstrap supports above $50 \%$ from 1000 replicates are shown. $\mathbf{c}$, $\mathbf{d}$ VrLFY gene expression was detected in SAM and developing leaf primordia. e The VrLFY sense probes were used as a negative control, and no hybridization signal was detected in SAM and leaf primordia. Scale bars $=100 \mu \mathrm{m}$

un in mungbean was allelic to $\operatorname{VrLFY}$, which encoded a putative plant-specific transcription factor closely related to UNI in pea and SGL1 in M. truncatula (Fig. 3b). In situ RNA hybridization data revealed that the $\operatorname{VrLFY}$ gene was expressed in the SAM and in the emerging leaf primordia (Fig. 3c) and showed relatively high expression in the distal portion of leaf primordia (Fig. 3d).

\section{Characterization and expression analysis of STM/BP-like $K N O X I$ genes in mungbean}

To characterize the KNOXI genes in mungbean, we conducted a BLAST search of its genome (http:// plantgenomics.snu.ac.kr), verifying candidate genes with the public transcriptome dataset ${ }^{30}$. Sixteen KNOX proteins were identified from mungbean and these were divided into three classes (class I, class II, and class M) based on phylogenetic analysis (Supplementary Figure 2$)^{30}$. Nine proteins were classified as STM-like KNOXI proteins (Vradi07g26830, Vradi10g07810 and Vradi06g03570), BP-like KNOXI protein (Vradi06g14320) and KNAT2/6-like KNOXI proteins (Vradi08g11380, Vradi03g07470, Vradi05g04350, Vradi11g09640, and Vradi0322S00070). Five KNOXII proteins were classified as

$$
\text { KNAT3/4/5-like }
$$

Vradi05g03240, and Vradi07g21010) and KNAT7-like proteins (Vradi11g02470 and Vradi07g13210). In addition, there were two members of class M KNOX proteins found in our mungbean sequence search (Vradi01g05360 and Vradi11g11780).

It has been reported that $S T M / B P$-like $K N O X I$ genes in tomato and $C$. hirsuta are expressed in the compound leaf and are involved in the control of lateral leaflet development ${ }^{4-6}$. However, STM/BP-like KNOXI genes are not associated with compound leaf development in M. truncatula and pea ${ }^{11-13}$. Previously, accumulation of KNOXI proteins in compound leaf primordia of nonIRLC legumes was detected by polyclonal KNOXI-specific antibodies $^{12}$. To compare the expression patterns of $S T M / B P$-like KNOXI genes in mungbean with those of IRLC-legume and model plant species, in situ RNA hybridization of four STM/BP-like KNOXI genes ( $\mathrm{ra}$ di07g26830, Vradi10g07810, Vradi06g03570, and Vradi06g14320) was carried out on sections of apices from 2week-old mungbean seedlings (Fig. 4). The results showed that there were different expression patterns among the four $S T M / B P$-like genes in mungbean (Fig. $4 \mathrm{a}-\mathrm{d}$ ). The expression of the two STM-like genes Vradi10g07810 and Vradi06g03570 was strongly detected in the shoot apical 

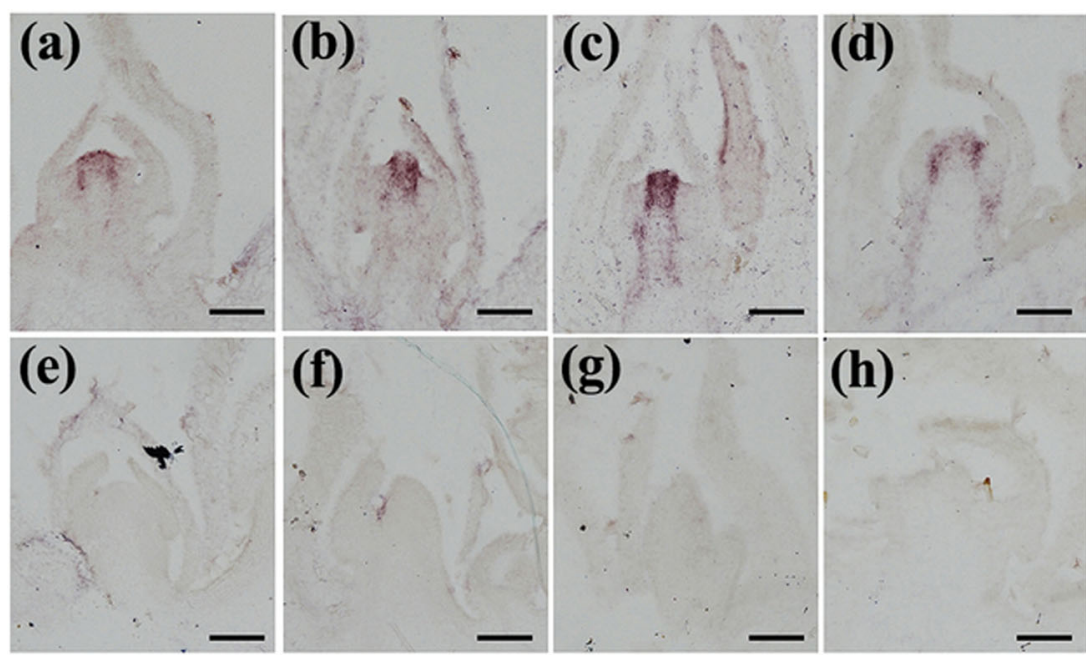

Fig. 4 RNA in situ hybridization analysis of STM/BP-like KNOXI gene expression in mungbean. RNA in situ hybridization analysis of a Vradi07g26830, b Vradi10g07810, c Vradi06g03570, and $\mathbf{d}$ Vradi06g14320 in the vegetative apices of mungbean. No expression was detected using the control sense probes of e Vradi07g26830, f Vradi10g07810, $\mathbf{g}$ Vradi06g03570, and $\mathbf{h}$ Vradi06g14320. Scale bars $=100 \mu \mathrm{m}$

meristem, and transcripts were also observed in the leaf primordia (Fig. 4b, c). However, expression of the STM-like gene Vradi07g26830 and the BP-like gene Vradi06g14320 was detected in the SAM but not in the leaf primordia (Fig. 4a, d).

Therefore, our data showed that the expression patterns of 2 STM-like KNOXI genes from mungbean differed from pea and M. truncatula, as in those species, no STMlike genes have been shown to be expressed in any stage of the compound leaf primordia ${ }^{13}$. Moreover, $B P$-like genes were not expressed in the compound leaf primordia of mungbean, $L$. japonicus, pea and M. truncatula, which is a different situation from the expression of these genes in C. hirsuta and tomato ${ }^{4,5,7,11,13,22}$.

\section{Transcript profiling of VrLFY downstream targets}

To address the molecular function of $V r L F Y$ during leaf development, the transcriptome of shoot apices of 2week-old seedlings from un1-1 mutant and wild-type plants was studied using RNA-Seq. A total of 538 differentially expressed genes (300 downregulated and 238 upregulated) were identified between the mutant and wild-type plants (Supplementary Table 2). The results revealed a significant representation of genes associated with circadian rhythm and plant hormone signal transduction (Supplementary Table 2). In the un1 mutants, the genes encoding proteins with high similarity to GIGANTEA 3 (GI3), GIGANTEA-like, Phytochrome A (PHYA), TIME OF CAB EXPRESSION 1 (TOC1), CIRCADIAN CLOCK ASSOCIATED 1 (CCA1), EARLY FLOWERING 3 (ELF3), and Adagio protein 3 (ADO3) were significantly downregulated, while those with high similarity to LATE ELONGATED HYPOCOTYL (LHY) were significantly upregulated. It has been reported that these are key factors in the regulation of the circadian clock and regulate important developmental transitions such as flowering, which was consistent with the involvement of LFY and its orthologs in controlling flowering time and phase transition $^{31-33}$.

Previous studies have reported that plant hormones, including auxin and gibberellins (GA), play critical roles in leaflet initiation and compound leaf development ${ }^{34,35}$. In our expression studies, auxin, GA, ethylene and cytokinin-related genes were significantly differentially expressed in mutants compared to wild-type plants (Supplementary Table 2). In addition, a number of receptor-like protein kinases were differentially expressed, which might imply that several signaling cascades involved in cell proliferation and differentiation play a significant role in the control of compound leaf development in mungbean.

The transcripts of the KNOX family genes showed no obvious differential expression (data not shown), with the exception that one of the class M KNOX genes, Vradi11g11780, was decreased by approximately eightfold in vegetative shoot apices of the un1-1 mutant compared with the wild-type plant (Supplementary Table 2), and this was confirmed by qRT-PCR (data not shown). It has been reported that increasing the expression of class M KNOX genes in Arabidopsis and tomato results in serrated leaves and a larger number of leaflets, respectively ${ }^{36,37}$.

\section{Genetic interactions affecting compound leaf development in mungbean mutants}

Other mutants that showed an increase in the number of leaflets were identified, including heptafoliate 

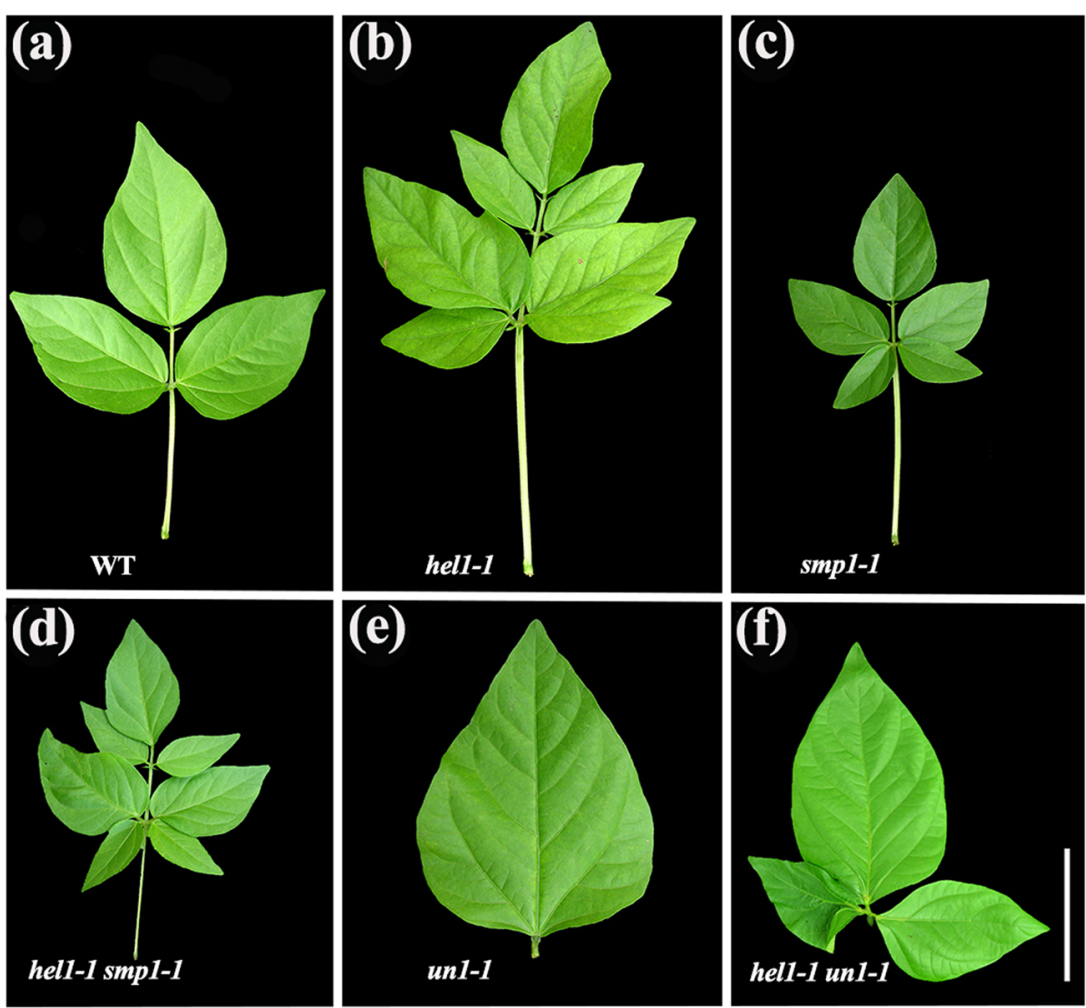

Fig. 5 Genetic interactions among leaf mutants in mungbean. Compound leaf phenotype of the wild type and mutants. a-f Mature compound leaves of a WT, b hel1-1, c smp1-1, d hel1-1 smp1-1, e un1-1, and f hel1-1 un1-1 mutants (all in the Sulu ecotype). Leaves of the hel1-1 un 1-1 double mutants exhibited three leaflets with short petioles. The hel1-1 smp1-1 double mutants were heptafoliate leaves of small size, indicating an epistatic interaction between hel1 and smp1 in the control of leaflet number. Scale bars $=10 \mathrm{~cm}$

leaflets1 (hel1) and small-pentafoliate leaflets1 (smp1), with 2 and 3 alleles, respectively (Fig. 5a-c; Supplementary Table 1). The juvenile leaves of the hel1 mutant showed an extreme dissection and leafletlike structures, sometimes associated with stipules, which developed in the proximal part of the blade (Supplementary Figure 3). The juvenile leaves of the $\operatorname{smp} 1$ mutant were normal, but the adult leaves exhibited five leaflets of small size (Fig. 5c). We made crosses between hel1 and smp1 to examine genetic interactions. In the resulting $F_{2}$ population, there were four classes of leaflet size and number: trifoliate, heptafoliate, smallheptafoliate, and small-pentafoliate. The numbers of plants in the different leaflet classes approximated a 9:3:3:1 ratio of the respective phenotypes (data not shown), suggesting that there were 2 unlinked genes controlling the multiple leaflet trait in mungbean. Further genetic analysis showed that the smallheptafoliate plants were hel1 smp1 double mutants (Fig. 5d), indicating that there was no additive phenotype of the double mutation in terms of leaflet number and that HEL1 interacted genetically with SMP1 in the control of leaflet number in mungbean compound leaf development.
To genetically test the involvement of $u n$ in the proliferation of lateral leaflet primordia in the hel1 mutant, we generated hel1-1 un1-1 double mutants. Juvenile plants of the double mutant were similar to that of a hell single mutant. However, all adult leaves in the hel1-1 un11 double mutants consisted of a short petiole bearing two lateral leaflets and one terminal leaflet (Fig. 5f), indicating the requirement for the $V r L F Y$ gene in the proliferation of lateral leaflet primordia in the hel1 mutant. In addition to the alterations in leaflet number, the hell and $u n$ mutants also exhibited alterations in the proximal-distal axis of compound leaves. Compared with wild-type leaves, the petiole length of mature leaves in the hel1 mutant was increased by approximately $10 \%$, while the mature leaves of the un mutant exhibited short petioles (Fig. 5b, e). Interestingly, the petiole length of mature leaves of hel1-1 un1-1 double mutants was short (Fig. 5f), resembling those of the $u n$ single mutant, suggesting that $u n$ is genetically epistatic to hel1 in leaf petiole development.

\section{HEL1 regulates the expression of $V r L F Y$ and $S T M / B P$-like KNOXI genes}

The genetic analysis described above indicated that HEL1 controls trifoliate development via 2 distinct 


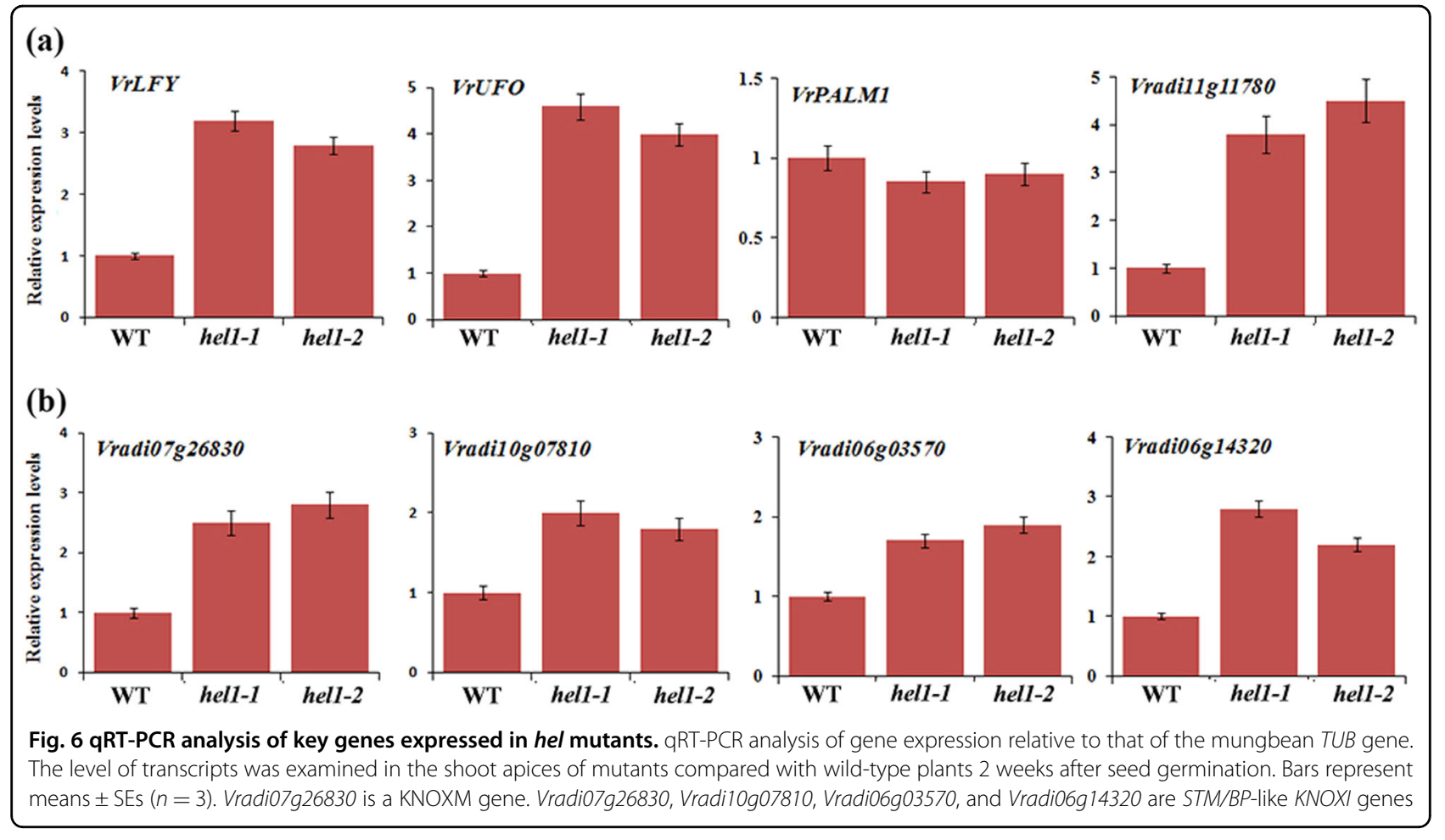

pathways, either dependent or independent of LFY. We first examined the expression of $V r L F Y$ and its putative upstream transcription factor PALM1 (VrPALM1) and cofactor UFO (VrUFO) in the wild type and hel1 mutants ${ }^{17,18}$. qRT-PCR data revealed that in vegetative shoot apices of the hel1 mutant compared with wild-type plants, $\operatorname{VrLFY}$ and $\operatorname{VrUFO}$ transcript levels were increased by threefold and fourfold, respectively (Fig. 6a). However, VrPALM1 transcript levels showed no obvious change in the hel1 mutant compared with wild-type plants. Because some KNOXI genes expressed in the compound leaf were likely associated with compound leaf development in mungbean, we further examined the expression of KNOX genes in the hel1 mutant and wild-type plants. The results showed that the expression of the $4 S T M / B P$-like KNOXI genes was significantly upregulated (Fig. 6b), while other KNOXI genes were not upregulated (data not shown). Interestingly, a KNOXM gene, Vradi11g11780 (Supplementary Figure 2), was also upregulated by fourfold in the hel1 mutant (Fig. 6a). Taken together, these data suggest that HEL1 regulates the expression of $L F Y$ and KNOXI genes to determine the leaflet number in mungbean.

\section{Discussion}

\section{The role of the $L F Y$ orthologs in compound leaf} development in legumes

In most compound-leafed species, activation of KNOXI gene expression in the leaf primordia is correlated with the development of compound leaves ${ }^{6,12}$. However, in
IRLC legume species with compound leaves such as pea and $M$. truncatula, the $S T M / B P$-like $K N O X I$ genes are excluded from the leaf primordia, and their expression is not correlated with compound leaf development ${ }^{11-13}$. In these plants, the $L F Y$ orthologs appear to function in place of the KNOXI genes in controlling compound leaf development ${ }^{12,15,16}$. The pea uni mutants and M. truncatula sgl1 mutants exhibit compound leaf defects, with all adult leaves changed to simple leaves, indicating that the $L F Y$ orthologs play a significant role in compound leaf development in IRLC legumes ${ }^{15,16}$.

The available information on the role of $L F Y$ orthologs in compound leaf development in non-IRLC legumes comes from phenotypic analysis of $L$. japonicus $p f m$ mutants and soybean $L F Y$ transgenic lines ${ }^{12,22}$. In the $p f m$ mutants, compound leaves lack 1 or 2 basal leaflets ${ }^{22}$. In transgenic soybean lines in which the endogenous $L F Y$ genes are downregulated, only the leaflet number of the compound leaves produced at the second node is reduced $^{12}$. In tomato, the mutant of the $L F Y$ ortholog, $f a$, has a reduced number of small leaflets present on the compound leaf ${ }^{32}$. Recently, it has been reported that the lfy mutant in C. hirsuta shows a lower number of leaflets than the wild type ${ }^{33}$. Therefore, it seems that the single leaflet phenotype caused by mutations of the $L F Y$ orthologs is only exhibited in IRLC legumes. It is thought that the $L F Y$ orthologs acquired a more significant role no earlier than the divergence of the Hologalegina clade from the other legumes ${ }^{12}$. 
However, our results showed that the un mutants in mungbean, with a complete conversion of compound leaves into simple leaves, were loss-of-function mutations of $V r L F Y$. This finding indicated that $V r L F Y$ could play a significant rather than a minor role in compound leaf development in mungbean, a member of the non-IRLC legumes (Fig. 2a-c). Interestingly, mutants exhibiting simple leaves and malformed flowers have also been reported in other non-IRLC legumes, including adzuki bean and cowpea ${ }^{38,39}$. While unproven, one could speculate that some of these mutant phenotypes in adzuki bean and cowpea, with similarities to the $u n$ mutants in mungbean, could also be caused by mutations of $L F Y$ orthologs. If so, $L F Y$ orthologs could play a significant role in compound leaf development in other non-IRLC legumes, not just in mungbean. In addition, the question of when in evolution $L F Y$ orthologs acquired a significant role in compound leaf development might need to be re-addressed ${ }^{12}$. Further investigation of the function of $L F Y$ orthologs in leaf development of different clades of non-IRLC legumes would be helpful in answering this question.

\section{Expression pattern of STM/BP-like KNOXI genes in legumes}

Phylogenetic analysis showed that there were duplications of KNOXI genes in legumes (Supplementary Figure 2). There was 1 STM gene and $1 B P$ gene in Arabidopsis, tomato and C. hirsuta. However, in IRLC legumes such as $M$. truncatula and pea, there were 2 $S T M$-like genes and $1 B P$-like KNOXI gene. In non-IRLC legumes such as mungbean and $L$. japonicus, there were 3 $S T M$-like genes and 1 BP-like KNOXI gene. In situ RNA hybridization showed that the 2 STM-like KNOXI genes in mungbean were expressed at the compound leaf primordia (Fig. 4), which was different from that of pea and $M$. truncatula in which none of the STM-like genes were expressed in any stage of the compound leaf primordia $^{11,13}$. BP-like genes were not expressed in the compound leaf primordia of mungbean (Fig. 4) and $L$. japonicus or in pea and $M$. truncatula $a^{5,7,9-11,13}$. This result was in contrast to findings of $B P$ orthologs from C. hirsuta and tomato, which were expressed in the compound leaf primordia ${ }^{25}$. It has been shown that differences in expression patterns between $B P$ from Arabidopsis and $C h B P$ from $C$. hirsuta are attributable to their cis-regulatory regions ${ }^{7}$. When KNOXI genes are overexpressed in M. truncatula and Alfalfa, there is an increase in leaflet number ${ }^{12,13}$. The loss of the role for KNOXI genes in compound leaf development of IRLC legumes also likely occurred due to a loss of expres$\operatorname{sion}^{12,13}$. However, this loss of expression of the STM-like and $B P$-like genes in compound leaf primordia in IRLC legumes could have occurred at different times in evolution because $B P$-like genes were also not expressed in compound leaf primordia in non-IRLC legumes, such as mungbean and L. japonicus.

\section{VrLFY could interact with KNOXI in mungbean to regulate compound leaf development}

In simple-leafed species, $L F Y$ orthologs play a key role in phase transition and floral development. Many downstream targets and DNA binding motifs of LFY have been identified at the genomic level in the model plant Arabidopsis $^{40,41}$. Despite its key role in compound leaf development in species such as pea and $M$. truncatula, how the LFY orthologs regulate downstream genes to affect lateral organ development, especially that of compound leaf development, remains elusive. In this study, transcriptomic analysis uncovered a total of 538 differentially expressed genes between mutants and the wild type. Several types of key factors, such as CCA1 and ELF3, involved in flowering time and phase transition were among the significantly differentially expressed genes (Supplementary Table 2), which was consistent with the conserved function of LFY orthologs in plants.

LFY has been reported to regulate the expression of some KNOXI genes such as BP and KNAT2 in Arabidopsis during pedicel and flower development ${ }^{41,42}$. However, the transcription levels of the KNOXI genes showed no obvious change in the un mutant compared with the wild-type plant (data not shown), suggesting that $\operatorname{VrLFY}$ does not regulate the expression of KNOXI genes at the transcriptional level in mungbean. Nevertheless, 1 class M KNOX gene, Vradi11g11780, with high similarity to PETROSELIUM (PTS) in tomato and FUSED COMPOUND LEAF (FCL2) in M. truncatula, was downregulated 3.5-fold in the un1-1 mutant ${ }^{37,43}$. It has been shown that the class M KNOX proteins in Arabidopsis and tomato could form heterodimers with BEL1-like homeodomain (BELL) proteins and interfere with the regulatory networks of KNOXI-BELL complexes during leaf development ${ }^{36}$. Transgenic Arabidopsis lines overexpressing KNATM-B exhibit serrated leaves, and a mutant with upregulated expression of the PTS gene in tomato exhibits a proliferation of compound leaves ${ }^{36,37}$. In M. truncatula, the class M KNOX gene FCL1 has been shown to control boundary establishment and petiole length of compound leaves and is required for the development of extra leaflet primordia in a palm1 mutant ${ }^{44}$. Therefore, our results indicated that $\operatorname{VrLFY}$ might modulate KNOXI regulatory networks by regulating the expression of a class M KNOX gene ( $\mathrm{rra}$ di11g11780) to control compound leaf development. Mutant databases for model legume plants such as M. truncatula and L. japonicus and legume crops such as soybean are available ${ }^{45-47}$. It would be worth identifying mutants of Vradi11g11780/FCL2 orthologs and dissecting their roles in compound leaf development in 
different legumes. Furthermore, it will also be necessary to identify mutations of the $S T M / B P$-like $K N O X I$ genes in mungbean and other non-IRLC legumes to investigate gene and protein interactions between KNOXI genes and the $L F Y$ orthologs during compound leaf development in non-IRLC plants.

\section{HEL1 could orchestrate VrLFY and KNOXI to control compound leaf development in mungbean}

Our results suggested that there were 2 distinct regulatory processes mediated by the LFY ortholog and KNOXI proteins during compound leaf development in mungbean. It also raised the question of how the two processes were coordinated during compound leaf development in mungbean. The HEL1 gene was a key locus of mungbean in the control of leaflet number, whose mutation resulted in dissected juvenile leaves and heptafoliate adult leaves (Fig. 5b and Supplementary Figure 3). Double mutant analysis showed that hel1 genetically interacted with $u n$ and $s m p 1$ to control the leaflet number of the compound leaves, indicating that lateral leaflet formation in the hel1 mutant was dependent not only on $L F Y$ but also on other regulators in the control of compound leaf development. Consistently, gene expression analysis showed that the $S T M / B P$-like $K N O X I$ genes and a $K N O X M$ gene, $\operatorname{VrLFY}$, as well as its putative cofactor $\operatorname{VrUFO}$, were significantly upregulated in the hel1 mutant compared to the wild type (Fig. 6). Therefore, these results suggested that HEL1 could coordinate the regulatory processes mediated by VrLFY and KNOXI to control compound leaf development in mungbean.

Interestingly, heptafoliate-leaf-like mutants similar to the hel1 mutant in mungbean were also identified and characterized in other legumes, including soybean and cowpea $^{48,49}$. In soybean, the seven-leaflet character is a single recessive trait conditioned by the lf 2 locus $^{45}$. Preliminary mapping results in mungbean revealed that the HEL1 gene was located to a region of chromosome 11, which showed synteny with the lf2 locus in soybean (data not shown $)^{50}$. Future work to clone the HEL1 gene and its ortholog in soybean and related legumes could provide new insights into the molecular mechanisms orchestrating the two regulatory processes mediated by the $L F Y$ ortholog and KNOXI genes during compound leaf development in mungbean and other legumes.

\section{Acknowledgements}

This work was supported by the National Natural Science Foundation of China (Grant No. 31700186) and the Ministry of Agriculture of China for Transgenic Research (Grant No. 2014ZX0800943B).

\section{Author details}

'Institute of Traditional Chinese Medicine and Natural Products, College of Pharmacy, Jinan University, Guangzhou, China. ${ }^{2}$ College of Life Sciences, Laboratory Center of Life Sciences, Nanjing Agricultural University, Nanjing, China. ${ }^{3}$ Guangdong Key Laboratory of Plant Resources, School of Life Sciences,
Sun Yat-Sen University, Guangzhou, China. ${ }^{4}$ Institute of Plant Stress Biology, State Key Laboratory of Cotton Biology, Department of Biology, Henan University, Kaifeng, China

Conflict of interest

The authors declare that they have no conflict of interest.

Publisher's note

Springer Nature remains neutral with regard to jurisdictional claims in published maps and institutional affiliations.

Supplementary Information accompanies this paper at (https://doi.org/ 10.1038/s41438-018-0088-0).

Received: 31 December 2017 Revised: 25 July 2018 Accepted: 7 August 2018

Published online: 01 February 2019

\section{References}

1. Long, J. A., Moan, E. I., Medford, J. I. \& Barton, M. K. A member of the KNOTTED class of homeodomain proteins encoded by the STM gene of Arabidopsis. Nature 379, 66-69 (1996).

2. Sinha, N. R., Williams, R. E. \& Hake, S. Overexpression of the maize homeo box gene, KNOTTED-1, causes a switch from determinate to indeterminate cell fates. Genes Dev. 7, 787-795 (1993).

3. Jackson, D., Veit, B. \& Hake, S. Expression of maize KNOTTED 1 related homeobox genes in the shoot apical meristem predicts patterns of morphogenesis in the vegetative shoot. Development 120, 405-413 (1994).

4. Hareven, D., Gutfinger, T., Parnis, A., Eshed, Y. \& Lifschitz, E. The making of a compound leaf: genetic manipulation of leaf architecture in tomato. Cell $\mathbf{8 4}$, 735-744 (1996).

5. Chen, J. J., Janssen, B. J., Williams, A. \& Sinha, N. A gene fusion at a homeobox locus: alterations in leaf shape and implications for morphological evolution. Plant Cell 9, 1289-1304 (1997).

6. Bharathan, G. et al. Homologies in leaf form inferred from KNOXI gene expression during development. Science 296, 1858-1860 (2002).

7. Hay, A. \& Tsiantis, M. The genetic basis for differences in leaf form between Arabidopsis thaliana and its wild relative Cardamine hirsuta. Nat. Genet. 38, 942-947 (2006).

8. Rast-Somssich, M. I. et al. Alternate wiring of a KNOXI genetic network underlies differences in leaf development of $A$. thaliana and C. hirsuta. Genes Dev. 29, 2391-2404 (2015)

9. Parnis, A. et al. The dominant developmental mutants of tomato, Mouse-ear and Curl, are associated with distinct modes of abnormal transcriptional regulation of a Knotted gene. Plant Cell 9, 2143-2158 (1997).

10. Shani, E. et al. Stage-specific regulation of Solanum lycopersicum leaf maturation by class 1 KNOTTED1-LIKE HOMEOBOX proteins. Plant Cell 21, 3078-3092 (2009).

11. Hofer, J., Gourlay, C., Michael, A. \& Ellis, T. H. Expression of a class 1 knotted1like homeobox gene is down-regulated in pea compound leaf primordia. Plant Mol. Biol. 45, 387-398 (2001).

12. Champagne, C. E. et al. Compound leaf development and evolution in the legumes. Plant Cell 19, 3369-3378 (2007).

13. Zhou, C. et al. STM/BP-like KNOXI is uncoupled from ARP in the regulation of compound leaf development in Medicago truncatula. Plant Cell 26, 1464-1479 (2014).

14. Weigel, D. Alvarez, J., Smyth, D. R. Yanofsky, M. F. \& Meyerowitz, E. M LEAFY controls floral meristem identity in Arabidopsis. Cell 69, 843-859 (1992).

15. Hofer, J. et al. UNIFOLIATA regulates leaf and flower morphogenesis in pea. Curr. Biol. 7, 581-587 (1997).

16. Wang, $\mathrm{H}$. et al. Control of compound leaf development by FLORICAULA LEAFY ortholog SINGLE LEAFLET1 in Medicago truncatula. Plant Physiol. 146, 1759-1772 (2008).

17. Taylor, S., Hofer, J. \& Murfet, I. Stamina pistilloida, the Pea ortholog of Fim and UFO, is required for normal development of flowers, inflorescences, and leaves. Plant Cell 13, 31-46 (2001). 
18. Chen, J. et al. Control of dissected leaf morphology by a Cys(2)His(2) zinc finger transcription factor in the model legume Medicago truncatula. Proc. Natl Acad. Sci. USA 107, 10754-10759 (2010).

19. Ge, L. \& Chen, R. (2014). PHANTASTICA regulates leaf polarity and petiole identity in Medicago truncatula. Plant Signal. Behav. 9, e28121 (2017).

20. Zhou, C. et al. The transacting short interfering RNA3 pathway and no apical meristem antagonistically regulate leaf margin development and lateral organ separation, as revealed by analysis of an argonaute7/lobed leaflet1 mutant in Medicago truncatula. Plant Cell 25, 4845-4862 (2013).

21. Peng, J., Berbel, A., Madueño, F. \& Chen, R. AUXIN RESPONSE FACTOR3 regulates compound leaf patterning by directly repressing PALMATE-LIKE PENTAFOLIATA1 expression in Medicago truncatula. Front. Plant Sci. 8, 1630 (2017).

22. Wang, Z. et al. Multiple components are integrated to determine leaf complexity in Lotus japonicus. J. Integr. Plant Biol. 55, 419-433 (2013).

23. Wang, Y. \& Chen, R. Regulation of compound leaf development. Plants 3, 1-17 (2013).

24. Chen, C., Wang, S. \& Huang, H. LEUNIG has multiple functions in gynoecium development in Arabidopsis. Genesis 26, 42-54 (2002).

25. Luo, J. H. et al. Different expression patterns of duplicated PHANTASTICA-like genes in Lotus japonicus suggest their divergent functions during compound leaf development. Cell Res. 15, 665-677 (2005).

26. Sairam, R. K., Dharmar, K., Chinnusamy, V. \& Meena, R. C. Water logginginduced increase in sugar mobilization, fermentation, and related gene expression in the roots of mung bean (Vigna radiata). J. Plant. Physiol. 166 602-616 (2009).

27. Mortazavi, A., Williams, B. A., McCue, K., Schaeffer, L. \& Wold, B. Mapping and quantifying mammalian transcriptomes by RNA-Seq. Nat. Methods 5, 621-628 (2008).

28. Audic, S. \& Claverie, J. M. The significance of digital gene expression profiles. Genome Res. 7, 986-995 (1997)

29. Sangsiri, C., Sorajjapinun, W. \& Srinivesc, P. Gamma radiation induced mutations in mungbean. ScienceAsia 31, 251-255 (2005).

30. Kang, Y. J. et al. Genome sequence of mungbean and insights into evolution within Vigna species. Nat. Commun. 5, 443-5451 (2014).

31. Shim, J. S., Kubota, A. \& Imaizumi, T. Circadian clock and photoperiodic flowering in Arabidopsis, CONSTANS is a hub for signal integration. Plant Physiol. 173, 5-15 (2017).

32. Molinero-Rosales, N. et al. FALSIFLORA, the tomato orthologue of FLORICAULA and LEAFY, controls flowering time and floral meristem identity. Plant J. 20, 685-693 (1999).

33. Monniaux, M. et al. Conservation vs divergence in LEAFY and APETALA1 functions between Arabidopsis thaliana and Cardamine hirsuta. New Phytol. 216, 549-561 (2017)
34. Hay, A. et al. The gibberellin pathway mediates KNOTTED1-type homeobox function in plants with different body plans. Curr. Biol. 12, 1557-1565 (2002).

35. Barkoulas, M., Hay, A., Kougioumoutzi, E. \& Tsiantis, M. A developmental framework for dissected leaf formation in the Arabidopsis relative Cardamine hirsuta. Nat. Genet. 40, 1136-1141 (2008).

36. Magnani, E. \& Hake, S. KNOX lost the OX, The Arabidopsis KNATM gene defines a novel class of KNOX transcriptional regulators missing the homeodomain. Plant Cell 20, 875-887 (2008).

37. Kimura, S., Koenig, D., Kang, J., Yoong, F. Y. \& Sinha, N. Natural variation in leaf morphology results from mutation of a novel KNOX gene. Curr. Biol. 18, 672-677 (2008).

38. Blakeslee, A. F. A unifoliolate mutation in adzuki bean. J. Hered. 4, 153-155 (1919).

39. Rawal, K. M., Porter, W. M., Franckowiak, J. D., Fawole, I. \& Rachie, K. O. Unifoliolate leaf, A mutant in cowpeas. J. Hered. 67, 193-194 (1976).

40. Moyroud, E. et al. Prediction of regulatory interactions from genome sequences using a biophysical model for the Arabidopsis LEAFY transcription factor. Plant Cell 23, 1293-1306 (2011).

41. Winter, C. M. et al. LEAFY target genes reveal floral regulatory logic, cis motifs, and a link to biotic stimulus response. Dev. Cell. 20, 430-443 (2011).

42. Yamaguchi, N., Yamaguchi, A., Abe, M., Wagner, D. \& Komeda, Y. LEAFY controls Arabidopsis pedicel length and orientation by affecting adaxial-abaxial cell fate. Plant J. 69, 844-856 (2012).

43. Di Giacomo, E. et al. KNAT3/4/5-like class 2 KNOX transcription factors are involved in Medicago truncatula symbiotic nodule organ development. New Phytol. 213, 822-837 (2017).

44. Peng, J. et al. Regulation of compound leaf development in Medicago truncatula by fused compoundleaf1, a class M KNOX gene. Plant Cell 23, 3929-3943 (2011)

45. Tadege, M. et al. Large scale insertional mutagenesis using Tnt1 retrotransposon in the model legume Medicago truncatula. Plant J. 54, 335-347 (2008).

46. Małolepszy, A. et al. The LORE1 insertion mutant resource. Plant J. 88, 306-317 (2016).

47. Li, Z. et al. Development and utilization of a new chemically-induced soybean library with a high mutation density. J. Integr. Plant Biol. 59, 60-74 (2017).

48. Fehr, W. R. Genetic control of leaflet number in soybeans. Crop Sci. 12 , 221-224 (1972)

49. Fawole, I. Genetic control of leaflet number and shape in cowpea, Vigna unguiculata (L) Walp. Niger. J. Sci. 34, 73-80 (2000).

50. Seversike, T. M., Ray, J. D., Shultz, J. L. \& Purcell, L. C. Soybean molecular linkage group B1 corresponds to classical linkage group 16 based on map location of the If (2) gene. Theor. Appl. Genet. 117, 143-147 (2008). 